\title{
The coming of age of microbial ecotoxicology: report on the first two meetings in France
}

\author{
Jean-François Ghiglione ${ }^{1,2,{ }^{*}, \text { Fabrice Martin-Laurent }}{ }^{3}$, Sabine Stachowski-Haberkorn ${ }^{4}$, \\ Stéphane Pesce ${ }^{5}$, Stéphane Vuilleumier ${ }^{6}$
}

\footnotetext{
${ }^{1}$ UMR 7621, Laboratoire d'Océanographie Microbienne, Observatoire Océanologique, CNRS, Avenue Fontaulé, 66650, Banyuls/mer, France

2 UPMC Univ Paris 06, UMR 7621, Laboratoire d'Océanographie Microbienne, Observatoire Océanologique, Sorbonne Universités, 66650, Banyuls/mer, France

${ }^{3}$ UMR 1347 Agroécologie, INRA, 17 rue Sully, 21065, Dijon, France

${ }^{4}$ Laboratoire d'écotoxicologie, rue de l'île d'Yeu, IFREMER, BP 21105, 44311, Nantes cedex 03, France

5 UR MALY, centre de Lyon-Villeurbanne, Irstea, 5 rue de la Doua-CS 70077, 69626, Villeurbanne cedex, France

${ }^{6}$ UMR 7156 UdS - CNRS Génétique Moléculaire, Génomique, Microbiologie, Université de Strasbourg, 67083, Strasbourg cedex, France
}

*: Corresponding author : Jean-François Ghiglione, email address : ghiglione@obs-banyuls.fr

\section{Context and objectives}

Microorganisms are ubiquitous in soil, air, and water ecosystems, where they are key players of ecosystem services. Microbial ecotoxicology is an emerging interdisciplinary area of research which aims at investigating the impact of human activities on the diversity, abundance, and activity of microorganisms. In return, the results of such investigations hold the promise to provide novel ways of assessing in a sensitive way the impacts of diverse environmental disturbances and subsequent ecosystem responses. Thus and although the term itself is yet rarely encountered in the scientific literature, microbial ecotoxicology already addresses an increasing political and societal demand. In the French scientific landscape, which often mimics the famous (but sometimes indigestible) "millefeuilles" pastry, microbial 
ecotoxicologists are scattered across many different research centers belonging to different research organizations and universities. This research field has thus lacked any visibility and remained unorganized until now. Formal organization of scientific activities may be considered a typical 'froggies' concern (or ailment). Nevertheless, it is rather surprising that scientific journals and significant international conferences specifically devoted to microbial ecotoxicology have been missing so far, especially considering the plethoric range of journals and congresses devoted to microbial ecology and ecotoxicology. With these considerations in mind, the idea of organizing the French research community of microbial ecologists around concepts of ecotoxicology made its way, with the aim of sharing the necessity to overcome artificial boundaries that prevent progress in this promising field.

\section{Coming out: the $1^{\text {st }}$ meeting on Microbial Ecotoxicology, Lyon-Villeurbanne, France, 20-}

\section{March 2013}

This meeting was organized by Drs Stéphane Pesce (Irstea UR MALY, Lyon-Villeurbanne) and Fabrice Martin-Laurent (INRA, UMR Agroécologie, Dijon) in March 2013 at Irstea in Lyon-Villeurbanne.

\section{Objectives}

The main objectives of this first meeting were i) to identify and bring together researchers, lecturers and professors in the field of microbial ecotoxicology, in order to establish an overview of the state of the art of research and teaching in microbial ecotoxicology in France, and ii) to initiate a collective brainstorming to identify future challenges of microbial ecotoxicology. The ultimate goals were to define and successfully implement a collective approach to design, evaluate and support investigations in the field of microbial ecotoxicology.

\section{Developing active participation in the research community: the "World Cafe" approach} A key challenge was to choose an optimal way to involve all potentially interested researchers in the process of defining the community of 'microbial ecotoxicologists'. The organizers decided to apply a participative approach coupled with a 'world cafe', a technique originally developed for meetings in which different interest groups confront their views and discuss common future activities (e.g. Jorgenson and Steier, J. Appl. Behav. Sc., 2013, 49:388-405). First, all potentially interested researchers were contacted before the workshop and asked to 
respond to an online questionnaire designed to provide an initial inventory of the strengths, weaknesses, wishes and development potential of microbial ecotoxicology, also taking the French and international research contexts into account.

Answering the questionnaire was a pre-requisite for effective inscription to the meeting. In total, 46 researchers and lecturers from 25 different laboratories affiliated to different institutes and universities in France responded to this solicitation, and 35 of them eventually participated to the workshop.

The results of this questionnaire as well as the results of bibliometric study on microbial ecotoxicology were presented to the participants of the workshop as an opening lecture, and provided the framework to develop subsequent exchanges. Keynote lectures were delivered by Drs Jeanne Garric (Irstea), Christian Mougin (INRA), Philippe Garrigues (CNRS) and Edward Topp (Agriculture and Agri-Food Canada), who all gave their respective vision about the main challenges facing ecotoxicology in the light of the worldwide environmental fate and dispersion of chemicals as a result of human activities.

Following these presentations and on the basis of the contextual elements that were identified, a collective prospective discussion followed, with three successive stages:
i) identification of the main stakes of microbial ecotoxicology;

ii) definition of objectives and associated courses of action;

iii) determination of the priority levels of actions.

In line with the chosen participative approach to promote interactive exchanges among all participants, moderation of the "World Café" was ensured by RCT Territoires (http://www.rct-territoires.com), a consulting business whose team designed and implemented structured conversational processes in which groups of researchers discussed a topic along several stations, with groups of researchers switching tables periodically and getting introduced to the previous discussion by a "station host". Giving their expertise in ecotoxicology, Drs Ed Topp and Philippe Garrigues acted as 'Witness Experts', moving from station to station to give advices and an external perspectives on the ongoing discussions.

\section{Main conclusions}

Four types of issues were defined:

i) social (i.e. how to promote interactions between research in microbial ecotoxicology and society?);

ii) scientific (i.e. how to better assess the effective biological and ecological "impact" of toxicants in complex environments?); 
iii) technology transfer (i.e. how to go from scientific knowledge to political decisions?);

iv) organizational (i.e. how to improve synergies between research groups and gain in visibility and readability?)

For each of them, concrete objectives were defined and courses of action were identified. Some of these actions were implemented quickly, thanks to the positive dynamics resulting from this workshop.

With regard to the organizational issue, the most concrete actions that were implemented were:

i) the setting-up of a website dedicated to microbial ecotoxicology (http://ecotoxicomic.irstea.fr), and of an associated mailing list to exchange information on microbial ecotoxicology (Fig. 1),

ii) the writing of a collectively reviewed and publicly available report on the microbial ecotoxicology website.

iii) the implementation of "microbial ecotoxicology" sessions in French congresses dedicated to ecotoxicology (The French Society of Ecotoxicology, SEFA, Thionville, July 2013), microbial ecology (The Francophone Association on Microbial Ecology, AFEM, Clermont-Ferrand, October 2013) and microbiology (The French Society of Microbiology, SFM, Institut Pasteur, Paris, April 2014),

iv) the scheduling of a first French scientific conference entirely devoted to Microbial Ecotoxicology (see below)

v) the implementation of 'microbial ecotoxicology' sessions in international congresses (XIVth International Congress of Bacteriology and Applied Microbiology, IUMS 2014, Montreal, Canada, July 27 - August 1, 2014)

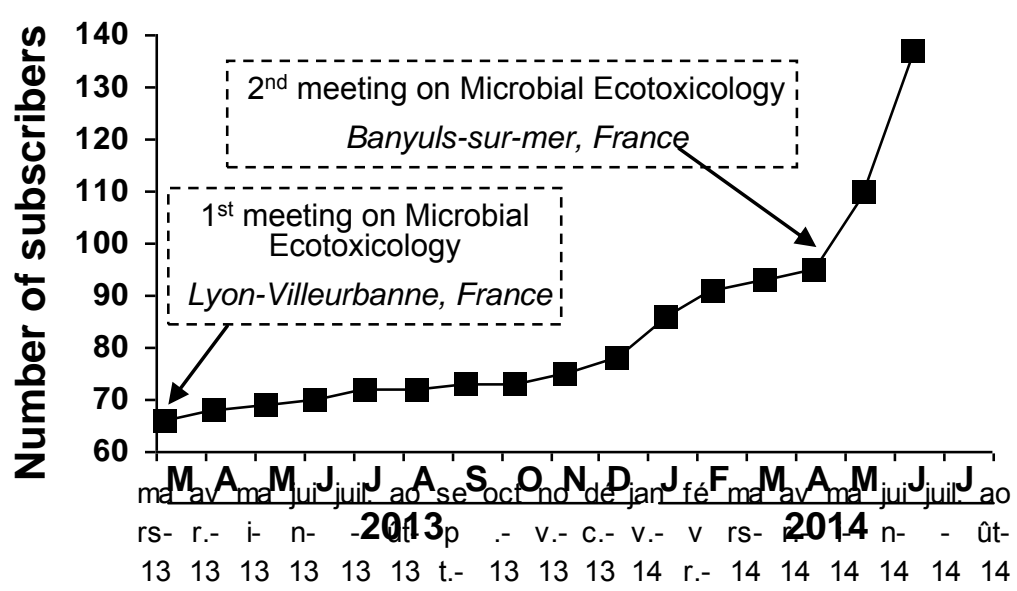

Fig. 1: Evolution of subsctibers to the "ectotoxicomic" mailing list 
From "World Café" to Science: the $2^{\text {nd }}$ meeting on Microbial Ecotoxicology, Banyuls-surmer, France, 21-23 May 2014

\section{Objectives and organization}

The $1^{\text {st }}$ prospective meeting on microbial ecotoxicology in Lyon in 2013 generated quite some interest in the microbial ecology, ecotoxicology and environmental chemistry fields, and prompted us to organize a scientific conference dedicated to the topic, to assess whether a community of microbial ecotoxicologists could emerge beyond day-to-day ad hoc contacts and collaborations between individual researchers.

This $2^{\text {nd }}$ meeting and scientific conference on microbial ecotoxicology was organized by $\mathrm{Dr}$ Jean-François Ghiglione (CNRS, Laboratoire d'Océanographie Microbienne LOMIC - UMR 7621) and took place in May 2014 at the Observatoire Océanologique de Banyuls, France.

This meeting was sponsored by the government research organizations in charge of the main national reference laboratories involved in microbial ecotoxicology, i.e. CNRS (National Center for Scientific Research), IFREMER (French Research Institute for Exploitation of the Sea), INRA (National Institute for Agronomic Research), IRD (Research and Development Institut) and Irstea (National Institute for Scientific and Technological Research for the Environment and Agriculture). The meeting was organized under the auspices of the Francophone Association for Microbial Ecology (AFEM), which aims to organize meetings in the field of microbial ecology (http://mio.pytheas.univ-amu.fr/AFEM). A scientific committee composed of the authors of the present report and of the AFEM administrative board validated the scientific program and abstracts, which are available on the conference website (http://www.obs-banyuls.fr/AFEM5).

In total, 70 researchers attended the meeting, and 36 scientific teams, coming from all over France, were represented (Fig. 2). Thus, the main goal of the meeting, i.e. to federate and organize the French community, was fulfilled. Most notably, $\mathrm{PhD}$ students and postdocs were in strong attendance although they had not been particularly targeted in meeting announcements. In our view, this testifies of the strong interest of the emerging generation of scientists for the interdisciplinary approach of microbial ecotoxicology.

The meeting included four sessions, with a total of 30 oral presentations, 10 poster contributions and 3 keynote lectures by Drs Philippe Garrigues, Jean-François Ghiglione, and Stéphane Pesce. On the first day, a round table moderated by the members of the scientific committee allowed initiating the discussion on possible ways of promoting and developing 
this specific scientific community. The four sessions were rather ambitious in their scope, 72 addressing most of the identified key questions in the field (see below for a detailed 173 description of each session). In order to avoid fragmentation of the community on the basis of 74 their preferred ecosystem, special care was taken to distribute reports on freshwater, marine water, sediment and soil ecosystems across the four sessions of the meeting.

Conference sessions and highlights

188 The first session dealt with aspects of the design and use of microbial bioassays, biomarkers,

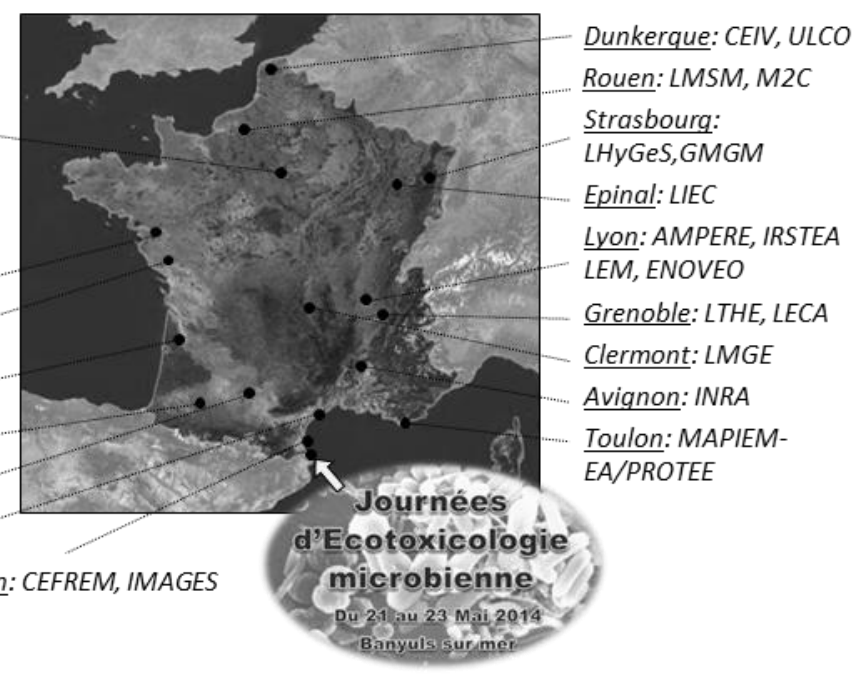

Fig. 2: Map showing the location (underlined) and the acronyms of the research units (in capitals) of presenters of oral presentations. An arrow from the logo of the meeting indicates the location of Banyuls-sur-mer. 
The second session addressed fundamental aspects of adaptation and resilience of microbial communities from a functional point of view. Although this often requires more comprehensive investigations and time investment than currently popular high-throughput sequencing approaches, functional investigations can be more easily integrated in models of ecosystem functioning, most notably the still somewhat neglected yet highly promising pollution-induced community tolerance (PICT) conceptual framework, and with often more rewarding results.

The third session was dedicated to this most classical of topics in environmental microbiology, bioremediation and biodegradation of contaminants. It was clearly symptomatic of the shift of focus in the field afforded by the emergence of an ecotoxicological perspective that this session attracted relatively few contributions compared to session 4 which, as described in more detail below, proposed a new, global and frontal take on the influence of pollutants, biotic and abiotic factors on microbial communities. Nevertheless, very exciting contributions were proposed in this session, both on long-standing environmental problems that urgently require satisfactory resolution, and on emerging and possibly still underestimated issues. On the former topic, for instance, novel insights on the biodegradation of chlordecone, an insecticide heavily used for decades in the French Antilles to treat banana plantations, and possibly the most recalcitrant chlorinated compound known, was reported. As a noted example on the latter subject, marked effects of exposure of soil communities to titanium oxide nanoparticles were found using a combination of functional assays and high-throughput sequence analysis.

The fourth session focused on the influence of pollutants, biotic and abiotic factors on microbial communities in the context of multiple stresses and contaminations. This topic attracted the most interest from conference applicants, resulting in a total of 17 presentations covering a wide range of environments, organisms and chemicals. Microbial communities that were investigated were not limited to bacteria, but also included archaea, fungi and microalgae. The aquatic (freshwater and marine) environment was the most popular, with studies dealing with microbial communities from water columns, biofilms and sediments, although several studies also addressed microbial communities from soil and the rhizosphere. Effects of various pollutants were assessed in a variety of experimental settings, such as laboratory microcosms and bioreactors, as well as in situ, in contaminated sites displaying different levels of a wide range of pollutants, including metals, polycyclic aromatic hydrocarbons, polychlorobiphenyls, pesticides, biocides and antibiotics. 
229 As in all studies dealing with field experiments, interpretation of results is often complicated

by numerous environmental variables that can, because of physical and chemical interactions that affect bioavailability of contaminants, and influence the toxicity of pollutants on organisms, and on microorganisms in particular. Nevertheless, working on microbial communities with relatively short generation times allows to more easily address key questions such as the extent to which contamination history (e.g., long-term, chronical, often low-level exposure versus short-term, high-level exposure) drives community structuring and successions in impacted ecosystems; and the resilience of ecosystems in the face of exposure to contaminants. In this context, this session has allowed to explicitly push forward the idea that not only individual organisms but also microbial communities can serve as bioindicators of the pollution-related ecological status of a given environment.

The data presented in this session also spelt out some commonly known but still unresolved issues. First of all, working with micro-organisms remains a challenge because of the high level of biological complexity associated with community level studies, and the resulting difficulties in data interpretation. For instance, a large proportion of phylotypes continue to lack physiologically characterized representatives, so that their roles, functions and interactions with the rest of the community remain unknown. The session made it clear that microbial ecotoxicology approaches may provide novel and important contributions to understand the effects of pollutants on the structure, function and dynamics of microbial communities. In particular, several contributions demonstrated how recent technological advances, particularly on molecular tools such as high-throughput sequencing, are providing decisive support. On the other hand, the need for sensitive but also robust approaches was also made evident. Indeed, techniques should be able to detect early disturbances at the level of global communities that i) can be measured in terms of function, and ii) cause detectable if even slight modifications of their structure. In both cases, however, the specific contribution of a given physico-chemical or specific biotic factor or organism to the "good state" or "good functioning" of the community will often be difficult to identify. Thus, key questions are still difficult to address at present, such as the definition of criteria to characterize disturbances of community structure and functions, and of microbe-based models to define normal operating range for microorganisms and to predict the risks of chemical contamination for ecosystem functioning through time, on different scales of size and in different geographical localizations. However, such questions were clearly central in current experimental designs of the participants of the meeting, so advances along these lines can reasonably be expected in the near future. 


\section{Conference outcomes}

This $2^{\text {nd }}$ meeting allowed us to gain a better insight on the forces in the field of microbial ecotoxicology in France, on specific research areas, and on the geography of the related network of laboratories involved in such research. Activities are clearly spread out all over the national territory and carried out by scientists belonging to research teams which generally do not identify microbial ecotoxicology as their core activity. Nevertheless, the feeling of belonging to a community was evident to all in the very rich discussions that took place all along the meeting. The wish of most participants to be a member of this community is also testified by the increase in the number of subscribers to the mailing list "ecotoxicomic" after the meeting in particular (Fig. 1).

A debate arose during the round table on the pertinence of creating a new of Francophone Microbial Ecotoxicologists, to facilitate organization of further meetings, sharing of knowledge and technical skills, and to make visible the vitality and the specificities of microbial ecotoxicology studies for colleagues and citizens. It was decided, as a concrete first outcome of the meeting, to take steps towards proposing and setting up a Pluridisciplinary Thematic Network (RTP) on microbial ecotoxicology to be hosted by the CNRS in conjunction with IFREMER, IRD, INRA, Irstea and other governmental research organizations for the next few years. In addition, the community of Microbial Ecotoxicologists is expected to reconvene in two years for the $3^{\text {rd }}$ meeting of Microbial Ecotoxicology, to be held in Clermont-Ferrand, France. Clearly, the field of microbial ecotoxicology has the potential to answer the wishes and needs of many scientists at the interface of microbial ecology, ecotoxicology and analytical chemistry, not only in France but also internationally. In this context, a special issue on microbial ecotoxicology will be proposed soon in ESPR in order to document this emerging field. We also strongly encourage authors of publications in the field to use the term "microbial ecotoxicology" as keyword, to increase the visibility of the international community of microbial ecotoxicologists.

\section{Acknowledgments}

The $1^{\text {st }}$ meeting was kindly funded by Irstea and by the INRA's national network of Ecotoxicologists. The $2^{\text {nd }}$ meeting greatly benefited from the support of our respective governmental research organizations (CNRS, IFREMER, INRA, IRD and Irstea) and of the Francophone Association for Microbial Ecology (AFEM). We would also like to thank the academic reception staff of Irstea in Lyon-Villeurbanne and of the Oceanology Observatory of Banyuls, as well as Anne-Laure Achard (Head of Scientific and Technical Information, Irstea Lyon-Villeurbanne) for bibliometric analysis. 